\title{
Electronic Structure Evolution across the Peierls Metal-Insulator Transition in a Correlated Ferromagnet
}

P. A. Bhobe, ${ }^{1,2,3}$ A. Kumar, ${ }^{4}$ M. Taguchi, ${ }^{1}$ R. Eguchi, ${ }^{1,2}$ M. Matsunami, ${ }^{1,2}$ Y. Takata, ${ }^{1,{ }^{*}}$ A. K. Nandy, ${ }^{4}$ P. Mahadevan ${ }^{4}$ D. D. Sarma, ${ }^{5}$ A. Neroni, ${ }^{6}$ E. Şaşıŏlu, ${ }^{6}$ M. Ležaić, ${ }^{6}$ M. Oura, ${ }^{1}$ Y. Senba, ${ }^{7}$ H. Ohashi, ${ }^{7}$ K. Ishizaka, ${ }^{2}$ M. Okawa, ${ }^{2}$ S. Shin, ${ }^{2,1}$ K. Tamasaku, ${ }^{1}$ Y. Kohmura, ${ }^{1}$ M. Yabashi, ${ }^{1}$ T. Ishikawa, ${ }^{1}$ K. Hasegawa, ${ }^{2}$ M. Isobe, ${ }^{2,8}$ Y. Ueda, ${ }^{2,9}$ and A. Chainani ${ }^{1, \dagger}$

${ }^{1}$ RIKEN SPring-8 Centre, 1-1-1 Sayo-cho, Hyogo 679-5148, Japan

${ }^{2}$ Institute for Solid State Physics, The University of Tokyo, Kashiwa, Chiba 277-8581, Japan

${ }^{3}$ Department of Physics, Indian Institute of Technology Indore, Indore 452 017, India

${ }^{4}$ Department of Condensed Matter Physics and Material Science,

S. N. Bose National Centre for Basic Sciences, Salt Lake, Kolkata-700098, India

${ }^{5}$ Solid State and Structural Chemistry Unit, Indian Institute of Science, Bangalore 560012, India

${ }^{6}$ PGI-1, Forschungszentrum Jülich D-52425 Jülich, and JARA, Germany

${ }^{7}$ JASRI/SPring-8, 1-1-1 Sayo-cho, Hyogo 679-5148, Japan

${ }^{8}$ Max Planck Institute for Solid State Research, Heisenbergstrasse 1, D-70569 Stuttgart, Germany

${ }^{9}$ Toyota Physical and Chemical Research Institute, Nagakute, Aichi 480-1192, Japan

(Received 28 October 2014; revised manuscript received 18 July 2015; published 9 October 2015)

Transition metal compounds often undergo spin-charge-orbital ordering due to strong electron-electron correlations. In contrast, low-dimensional materials can exhibit a Peierls transition arising from low-energy electron-phonon-coupling-induced structural instabilities. We study the electronic structure of the tunnel framework compound $\mathrm{K}_{2} \mathrm{Cr}_{8} \mathrm{O}_{16}$, which exhibits a temperature-dependent ( $T$-dependent) paramagnetic-toferromagnetic-metal transition at $T_{C}=180 \mathrm{~K}$ and transforms into a ferromagnetic insulator below $T_{\mathrm{MI}}=95 \mathrm{~K}$. We observe clear $T$-dependent dynamic valence (charge) fluctuations from above $T_{C}$ to $T_{\mathrm{MI}}$, which effectively get pinned to an average nominal valence of $\mathrm{Cr}^{+3.75}\left(\mathrm{Cr}^{4+}: \mathrm{Cr}^{3+}\right.$ states in a 3:1 ratio) in the ferromagnetic-insulating phase. High-resolution laser photoemission shows a $T$-dependent BCS-type energy gap, with $2 G(0) \sim 3.5\left(k_{B} T_{\mathrm{MI}}\right) \sim 35 \mathrm{meV}$. First-principles band-structure calculations, using the experimentally estimated on-site Coulomb energy of $U \sim 4 \mathrm{eV}$, establish the necessity of strong correlations and finite structural distortions for driving the metal-insulator transition. In spite of the strong correlations, the nonintegral occupancy $(2.25 d$-electrons/Cr) and the half-metallic ferromagnetism in the $t_{2 g}$ up-spin band favor a low-energy Peierls metal-insulator transition.

DOI: 10.1103/PhysRevX.5.041004

\section{INTRODUCTION}

The electron-correlation-driven metal-to-insulator transition (MIT) remains a central problem in condensedmatter physics. A great deal of our understanding of MITs is based on the concepts of the Mott transition and the Hubbard model [1,2]. $\mathrm{V}_{2} \mathrm{O}_{3}$ is considered to be the best realization of Mott-Hubbard physics, with a first-order transition from a paramagnetic metal to an antiferromagnetic (AFM) insulator [3]. However, recent studies have highlighted a ferromagnetic (FM)-insulating ground state in some strongly correlated transition-metal compounds.

\footnotetext{
* Deceased.

†chainania@gmail.com
}

Published by the American Physical Society under the terms of the Creative Commons Attribution 3.0 License. Further distribution of this work must maintain attribution to the author(s) and the published article's title, journal citation, and DOI.
Subject Areas: Condensed Matter Physics

Prominent examples include $\mathrm{La}_{2} \mathrm{NiMnO}_{6}$ [4-6], strained films of $\mathrm{SrVO}_{3}$ [7], and Cr-doped $\mathrm{VO}_{2}$ [8]. The discovery of a FM-metal-to-FM-insulator transition in $\mathrm{K}_{2} \mathrm{Cr}_{8} \mathrm{O}_{16}$ [9] provides a novel and unique case to study the role of strong correlations coupled to a Peierls transition while maintaining $\mathrm{FM}$ spin order.

$\mathrm{K}_{2} \mathrm{Cr}_{8} \mathrm{O}_{16}$ is a paramagnetic metal at room temperature, which transforms to a FM metal below $T_{C}=180 \mathrm{~K}$ [9]. The MIT takes place at a lower temperature, $T_{\mathrm{MI}}=95 \mathrm{~K}$, while retaining the FM order. While the rise in electrical resistivity below $95 \mathrm{~K}$ indicated a MIT, a simple activation energy could not be extracted from the resistivity data. The FM-insulating state showed a large saturation magnetization with an effective magnetic moment of $17.7 \mu_{B} / 8 \mathrm{Cr}$. This value of the magnetic moment can be viewed to arise from a mixture of formally $\mathrm{Cr}^{4+}$ and $\mathrm{Cr}^{3+}$ states in a $3: 1$ ratio (average valency $\mathrm{Cr}^{3.75} ; 2.25 d$-electrons/ $\mathrm{Cr}$ ), consistent with its chemical formula [9]. Interestingly, no hysteresis was observed in the $T$ dependence of resistivity, 
suggesting inapplicability of a first-order transition [9]. First-principles electronic structure calculations using the generalized gradient approximation (GGA) predicted the formation of an incommensurate long-wavelength charge density wave (CDW) caused by Fermi-surface nesting in a half-metallic system, which could lead to a gap formation in the majority spin band of $\mathrm{Cr} 3 d$ states [10]. The FM order in this case arises from a double-exchange-type mechanism. In contrast, GGA $+U$ (where $U$ is the on-site Coulomb energy) calculations predicted a weak charge disproportionation and an insulating ground state induced by $U$, but with a polaronic distortion of the crystal structure [11]. Here, it is clarified that the charge disproportionation corresponds to different occupancies of the majority spin states, which are suitably compensated by the different occupancies of the minority spin states, while the net charge essentially changes by only a very small fraction, typically $0.05-0.10 d$-electron count. These changes support a weak charge disproportionation and is very similar to results known for the manganites [12,13]. A recent firstprinciples study on transition-metal compounds (including titanates, manganites, and ferrites) showed that if the system is strongly hybridized, then a formal change of the oxidation state is compensated by a lowering of the ligand energy states. In such a situation, doping leads to ligand-character holes and a very small change in $d$ electron count [14]. Recent synchrotron-radiation-based diffraction studies have indeed detected the presence of weak superstructure reflections below $95 \mathrm{~K}$ that gradually grew in intensity with decreasing temperature $T$, indicative of a second-order Peierls transition [15,16]. Based on $\mathrm{GGA}+U$ calculations, it was proposed that $\mathrm{K}_{2} \mathrm{Cr}_{8} \mathrm{O}_{16}$ undergoes a quasi-1D "pure" Peierls instability of fully spin-polarized electrons at the Fermi level $\left(\mathrm{E}_{F}\right)$, giving rise to a MIT where electron correlations essentially play no role [15]. In contrast, a very recent GGA $+U$ study has claimed that $\mathrm{K}_{2} \mathrm{Cr}_{8} \mathrm{O}_{16}$ exhibits a Mott-Peierls transition [17]. However, it is noted that a Mott-Peierls transition is expected to show spectral changes in the $3 d$ density of states (DOS) over large energy scales of the order of the on-site Coulomb energy $U$, as is well known from the case of $\mathrm{VO}_{2}$ [18-20]. We experimentally answer this question in the present work. In this context, it is also important to clarify the terminology used in the literature: A "Mott-Peierls transition" is also called a "correlation-assisted Peierls transition"; i.e., the emphasis is on the Peierls transition [17-20]. This is different from the term "Peierls-Mott transition," which is used to describe a "Peierls-assisted orbital-selective Mott transition" with the emphasis on the Mott transition [21]. The difference is characterized by the low-frequency behavior of the on-site component of the selfenergy, which is linear for the Mott-Peierls case and diverges for the Peierls-Mott case [18,21].

The original Peierls transition arising from a structural distortion in a quasi-1D metal involves the development of a $T$-dependent superstructure, i.e., a periodic modulation of the charge density [22,23]. The Peierls transition leads to the formation of a Bardeen-Cooper-Schrieffer (BCS)-like $T$-dependent low-energy scale gap given by $2 G(0)=$ $3.5\left(k_{B} T_{\mathrm{MI}}\right)$ [23-25]. In contrast, it is known that the isostructural $\mathrm{K}_{2} \mathrm{~V}_{8} \mathrm{O}_{16}$ [26] undergoes a first-order MIT at $170 \mathrm{~K}$ with the possible formation of $\mathrm{V}^{4+}-\mathrm{V}^{4+}$ and $\mathrm{V}^{3+}-\mathrm{V}^{3+}$ pairs in the low- $T$ insulating phase. Also, $\mathrm{Rb}_{2} \mathrm{~V}_{8} \mathrm{O}_{16}$ undergoes a MIT with a discontinuity in resistivity indicating a first-order transition [27]. In this context, it is important to address the role of crystal structure in these materials. These materials exhibit the hollandite structure and are characterized by a tunnel framework structure, as shown in Fig. 1. With the general formula $\mathrm{A}_{2} \mathrm{M}_{8} \mathrm{O}_{16}$ (M-transition metal), the $\mathrm{MO}_{6}$ octahedral units form edge-sharing double chains, and four such chains are linked at the corners to form large tunnels running along the $c$ axis. The A-cation sits in the hollows of these tunnels. An unusual hybridization between the transition metal $3 d$ and oxygen $2 p$ states may be expected because of the presence of both edge-sharing and cornersharing $\mathrm{MO}_{6}$ octahedra, leading to an electronic structure that gives rise to unique properties [9-11,15]. From a detailed study [16] of the crystal structure at $T=150 \mathrm{~K}$ and $20 \mathrm{~K}$, it was shown that $\mathrm{K}_{2} \mathrm{Cr}_{8} \mathrm{O}_{16}$ exhibits a structural change from a tetragonal high- $T$ phase to a monoclinic low$T$ phase. From a valence-bond analysis, it was concluded that the $\mathrm{Cr}$ valency was the same for all $\mathrm{Cr}$ atoms within experimental errors $(<0.03$ electrons/Cratom $)$ for the high- $T$ and low- $T$ phases. This result indicates the absence of a static charge order of $\mathrm{Cr}$ atoms with different valencies

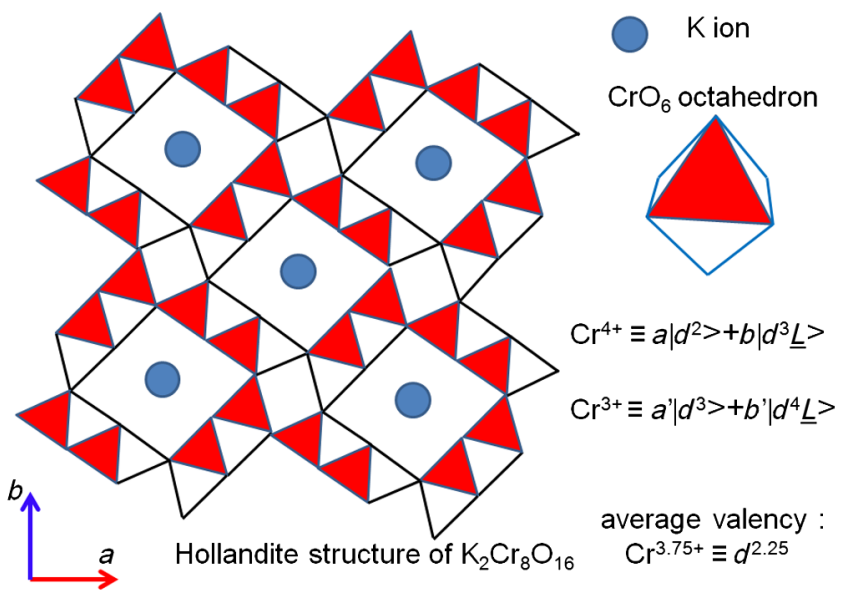

FIG. 1. The tunnel framework structure of the hollandite $\mathrm{K}_{2} \mathrm{Cr}_{8} \mathrm{O}_{16}$. As seen in the $a b$ plane, the hollandite structure consists of tunnels made out of double chains of $\mathrm{CrO}_{6}$ octahedra, stacked along the $c$ axis. The average valency of $\mathrm{Cr}$ ions is $\mathrm{Cr}^{3.75+}$, which formally corresponds to $\mathrm{Cr}^{4+}\left(d^{2}\right)$ and $\mathrm{Cr}^{3+}\left(d^{3}\right)$ states in a $3: 1$ ratio $(2.25 d$ electrons $/ \mathrm{Cr})$. The ground state has comparable contributions from $d^{n} \underline{L}^{0}\left(\equiv d^{n}\right)$ and $d^{n+1} \underline{L}^{1}$ ( $\underline{L}^{1}$ : ligand hole) for the $\mathrm{Cr}^{3+}$ and $\mathrm{Cr}^{4+}$ states. 
[16], although the chemical formula of $\mathrm{K}_{2} \mathrm{Cr}_{8} \mathrm{O}_{16}$ corresponds to a nonintegral valency of $\mathrm{Cr}^{+3.75}[9,15]$.

In view of the complex behavior emerging from earlier studies and still being debated, we felt it important to carry out spectroscopic studies of $\mathrm{K}_{2} \mathrm{Cr}_{8} \mathrm{O}_{16}$ and compare them with first-principles band-structure calculations in order to characterize its experimental electronic structure. In particular, we examine the evolution of the electronic structure as it transforms from a paramagnetic metal to a FM metal phase, and then into a FM insulator in $\mathrm{K}_{2} \mathrm{Cr}_{8} \mathrm{O}_{16}$. We report a combination of hard $\mathrm{x}$-ray, soft $\mathrm{x}$-ray and laser photoemission spectroscopy (PES), x-ray absorption spectroscopy (XAS), as well as GGA $+U$ and parametrized Hartee-Fock $(\mathrm{HF})+U$ calculations for $\mathrm{K}_{2} \mathrm{Cr}_{8} \mathrm{O}_{16}$. Resonant PES (res-PES) is used to identify the $\mathrm{Cr} 3 d$ character partial DOS at the Fermi level $\left(\mathrm{E}_{F}\right)$. Res-PES also shows the presence of a $\mathrm{Cr} 3 d$ two-hole satellite and provides an estimate of $U \sim 4.2 \mathrm{eV}$, consistent with the value of $U$ extracted from a constrained random-phaseapproximation (RPA) approach [28-31]. Hard x-ray PES (HAXPES)[32,33] shows a $T$-dependent valence evolution at high $T$ which stops changing in the Peierls-insulating phase. Laser-PES [34] shows a $T$-dependent opening of the gap, which is consistent with a BCS-type transition and rules out a Mott-Peierls transition. While the obtained large $U$ causes a considerable depletion of $\mathrm{Cr} 3 d$ character states at $\mathrm{E}_{F}$, it is not the sole factor to drive the system into the insulating phase. Our GGA $+U$ and $\mathrm{HF}+U$ calculations prove that a small local structural distortion, in addition to large $U$, is necessary to drive $\mathrm{K}_{2} \mathrm{Cr}_{8} \mathrm{O}_{16}$ into the FMinsulating phase. In the absence of structural distortions, GGA $+U$ calculations indicate a metallic state at least up to a $U$ of $4 \mathrm{eV}$. The results indicate that strong correlations set up the large-energy-scale electronic structure, while the structural distortion causes a weak-coupling BCS-like transition within about $35 \mathrm{meV}$ of $\mathrm{E}_{F}$ in $\mathrm{K}_{2} \mathrm{Cr}_{8} \mathrm{O}_{16}$.

\section{EXPERIMENTAL DETAILS}

Polycrystalline $\mathrm{K}_{2} \mathrm{Cr}_{8} \mathrm{O}_{16}$ used in the present study was prepared by a high-pressure synthesis and characterized for its crystal structure, electrical, and magnetic properties, as was reported in Ref. [9]. HAXPES measurements were carried out at beamline BL29XU, SPring-8 using an incident photon energy of $h \nu=7935 \mathrm{eV}$ and a spectrometer [32,33] equipped with a R-4000-10 kV VG-Scienta analyzer. The total energy resolution was set to $225 \mathrm{meV}$ for the HAXPES measurements. The soft x-ray PES was performed at BL17SU, at SPring-8 using a GammadataScienta SES2002 hemispherical electron analyzer set to the total energy resolution of $200 \mathrm{meV}$. XAS was recorded in the total electron yield mode. Laser PES was performed using the R4000WAL electron analyzer and a vacuumultraviolet laser $(h \nu=6.994 \mathrm{eV}, \Delta E=5 \mathrm{meV}$ at $10 \mathrm{~K})$ [34] at ISSP, University of Tokyo. All measurements were carried out in vacuum below $4 \times 10^{-8} \mathrm{~Pa}$, and clean sample surfaces were obtained by cleaving in situ at low $T$. The spectra were calibrated using the $E_{F}$ measured from a gold film evaporated onto the sample holder. $T$-dependent measurements were carried out using a liquid He flowtype cryostat and were checked for reproducibility on $T$ cycling.

\section{RESULTS AND DISCUSSIONS}

\section{A. Valence-band photoemission}

Figure 2(a) presents the valence-band (VB) spectrum of $\mathrm{K}_{2} \mathrm{Cr}_{8} \mathrm{O}_{16}$ at $20 \mathrm{~K}$ (insulating phase) and $140 \mathrm{~K}$ (metallic phase) obtained using soft $\mathrm{x}$ rays $(h \nu=1200 \mathrm{eV})$. The spectrum shows a peak located at $1.2 \mathrm{eV}$ binding energy (BE) and broad features at 4-8 eV BE. On comparing with known band-structure calculations [10,11], the feature at $1.2 \mathrm{eV} \mathrm{BE}$ is assigned to $\mathrm{Cr} 3 d$ states, while the broad features at about 4-8 eV BE are dominantly $\mathrm{O} 2 p$ states. We confirm the $\mathrm{Cr} 3 d$ orbital-selective partial DOS by VB res-PES across the $\mathrm{Cr} 2 p-3 d$ (L-edge) absorption threshold. These spectra are presented in Fig. 2(c), and the actual incident photon energies are indicated by tick marks on the $\mathrm{Cr} L_{3}$ XAS curve shown in the inset. Accordingly, Fig. 2(b) shows the VB spectrum obtained on resonance $(h \nu=578.05 \mathrm{eV})$, and the remarkable increase in the intensity of the $1.2-\mathrm{eV}$ peak confirms its $\mathrm{Cr} 3 d$ character. The tail of this $\mathrm{Cr} 3 d$ peak reaches $\mathrm{E}_{F}$ (see Fig. 2), indicating a highly reduced DOS at $\mathrm{E}_{F}$ in GGA $+U$ calculations [11] compared to GGA calculations [10], but it undergoes a metal-to-insulator transition with a small energy gap (about $35 \mathrm{meV}$ ) only in the presence of strong

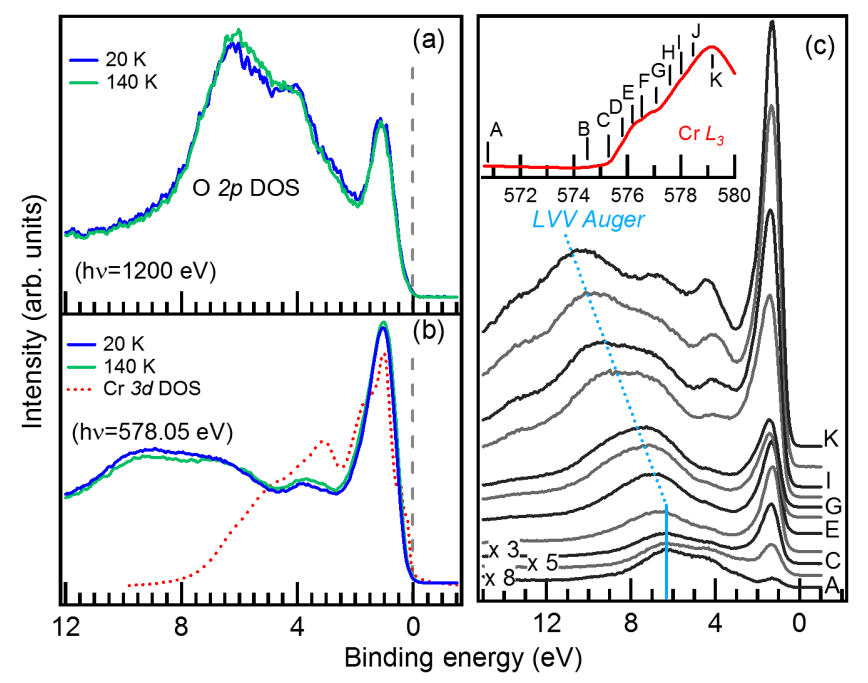

FIG. 2. Soft X-ray valence-band photoemission spectroscopy (PES) of $\mathrm{K}_{2} \mathrm{Cr}_{8} \mathrm{O}_{16}$. Valence band PES spectra of $\mathrm{K}_{2} \mathrm{Cr}_{8} \mathrm{O}_{16}$ obtained using (a) $h \nu=1200 \mathrm{eV}$ and (b) $h \nu=578.05 \mathrm{eV}$ and compared with the calculated Cr $3 d$ partial DOS. (c) ResonantPES measured across the $\mathrm{Cr} 2 p-3 d$ threshold at $20 \mathrm{~K}$ using photon energies indicated by tick marks (A-K) on the $\mathrm{Cr} L_{3} \mathrm{XAS}$ curve shown in the inset. 
correlations and a structural distortion, as discussed in detail later. Interestingly, as seen in Figs. 2(a) and 2(b), the $\mathrm{Cr} 3 d$ states show negligible changes between $20 \mathrm{~K}$ (insulating phase) and $140 \mathrm{~K}$ (metallic phase) on the energy scale of about 1-2 eV, indicating a clear difference with the behavior known for $\mathrm{VO}_{2}$, a prototypical Mott-Peierls system [18-20].

As the excitation energy is increased from $\mathrm{A}$ to $\mathrm{D}$, the intensity of a feature at about $6 \mathrm{eV}$ BE gets enhanced along with the $\mathrm{Cr} 3 d$ main peak, as shown in Fig. 2(c). This satellite feature evolves into an Auger feature $\left(L V V_{\text {Auger }}\right)$ at higher excitation energies, and it tracks the photon energy, as indicated by a dotted line. In such a situation, $U$ can be estimated by the equation $U=E_{2 p}-\left[h \nu-\mathrm{BE}_{\text {Auger }}\right]-2 \epsilon_{3 d}$, where $E_{2 p}$ corresponds to the $\mathrm{BE}$ of the $\mathrm{Cr} 2 p$ main peak, $h \nu$ is the incident photon energy, and $\mathrm{BE}_{\text {Auger }}$ is the $\mathrm{BE}$ of the corresponding Auger peak. Here, $2 \epsilon_{3 d}$ is the average energy of two uncorrelated holes, obtained from the selfconvolution of the one-electron removal $\mathrm{Cr} 3 d$ main peak. We estimate $U \sim 4.2 \mathrm{eV}$ using the experimental values of $\left[h \nu-\mathrm{BE}_{\text {Auger }}\right] \sim 567.9 \mathrm{eV}, 2 \epsilon_{3 d}=2.4 \mathrm{eV}$, and $E_{2 p}=$ $574.5 \mathrm{eV}$ obtained from $\mathrm{Cr} 2 p$ core-level PES (see Ref. [35], Fig. S5). A similar analysis has been carried out for $\mathrm{Cr}$ metal [36], $\mathrm{Cr}$ silicide [37], $\mathrm{CrN}$ [38], and $\mathrm{CaCrO}_{3}$ [39], indicating comparable values of $U$. We have self-consistently calculated the Hubbard $U$ and Hund exchange $J$ values by employing the constrained RPA approach [28] within the full-potential linearized augmented plane-wave (FLAPW) method [29] using Wannier functions [30,31]. The obtained $U$ and $J$ values for localized $\mathrm{Cr} 3 d$ orbitals are $3.4 \mathrm{eV}$ and $0.66 \mathrm{eV}$, respectively.

\section{B. First-principles band-structure calculations}

In order to elucidate the role of a large $U$ on the electronic structure of $\mathrm{K}_{2} \mathrm{Cr}_{8} \mathrm{O}_{16}$, we have carried out first-principles band-structure calculations using the GGA $+U$ and $\mathrm{HF}+U$ methods (see Ref. [35], Method 1 for details). We first carried out GGA $+U$ calculations within a plane-wave-projected augmented-wave implementation of density functional theory within VASP $[40,41]$. The experimental crystal structure [16] showing the tetramerization (Ref. [35], Fig. S1) has been considered, and the internal coordinates have been optimized using a $U$ of $4 \mathrm{eV}$. A $k$-points mesh of $4 \times 4 \times 16$ points was used and found to be adequate, as increasing the number of $k$-points to $6 \times 6 \times 18$ changed the total energy by less than $1 \mathrm{meV}$ per formula unit. A plane-wave cutoff of $400 \mathrm{eV}$ was used for the calculations. The results of GGA $+U$ calculations carried out with $U$ set to $4 \mathrm{eV}$, as estimated from the res-PES analysis, are shown in Fig. 3 for the obtained spin and atom-projected DOS. The results of Fig. 3(a), obtained for the high-temperature experimental crystal structure $[9,15,16]$, correctly indicate a metallic phase. On introducing a structural distortion, as obtained for the
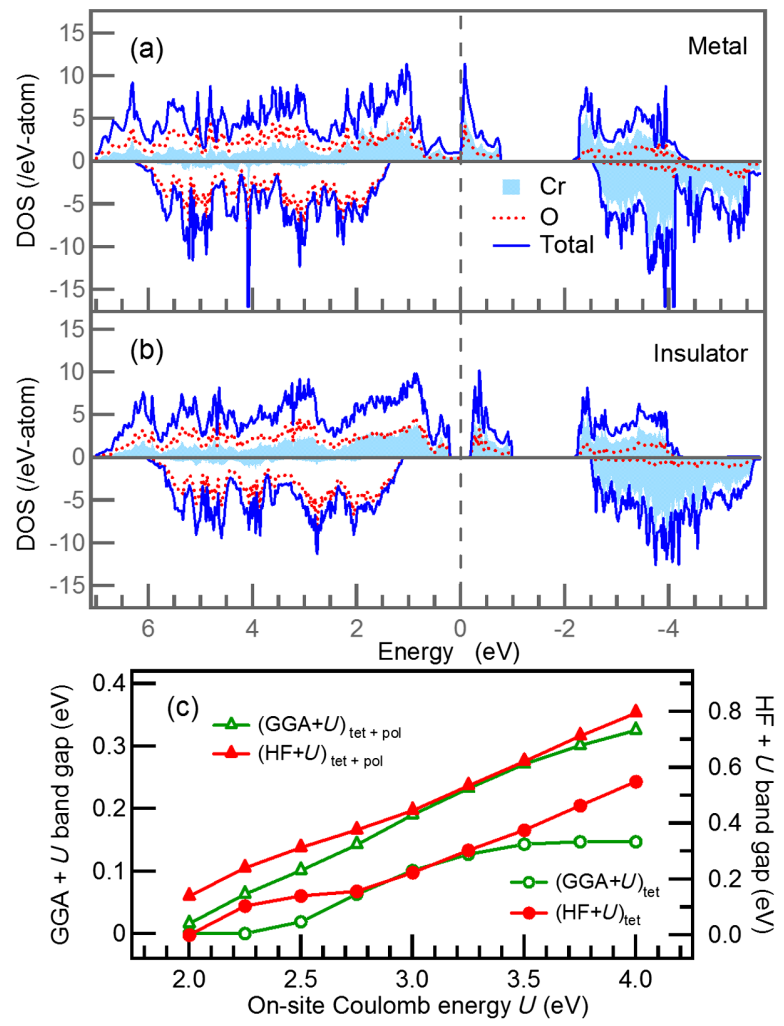

FIG. 3. DOS and band gap from first-principles calculations. (a) The atom and spin-projected DOS calculated using GGA $+U$ $(U=4 \mathrm{eV})$ gives a metallic phase for the high-temperature structure. (b) Introducing a structural distortion in the presence of $U$ opens up a gap at zero energy $\left(\mathrm{E}_{F}\right)$ (see text for details). (c) Band gaps calculated using $\mathrm{HF}+U$ and GGA $+U$ methods for different values of $U$, assuming a structural tetramerization (tet) without and with polaronic (pol) distortions.

low-temperature structure indicative of tetramerization with a $\sqrt{2} a \times \sqrt{2} a \times c$ unit cell $[15,16]$, or a symmetrybreaking polaronic distortion within the $a \times a \times c$ unit cell of the metal phase, an insulating gap opens up within the $t_{2 g}$ manifold of the DOS. The calculated DOS in the metallic and insulating phases [Figs. 3(a) and 3(b), respectively] are quite similar to recent $\mathrm{GGA}+U$ calculations with $U=$ $3 \mathrm{eV}[15,17]$. As is also observed in experimental VB spectra, the VB features of the calculated DOS seem very similar in the two phases, and no major changes are seen over large energy scales [Figs. 2(a) and 2(b)]. Moreover, the Cr $3 d$ partial DOS convoluted with a Gaussian function (corresponding to the experimental resolution) shows an excellent match with the on-resonance VB spectrum presented in Fig. 2(b). Similarly, the unoccupied $\mathrm{Cr} 3 d$ and $\mathrm{O} p$ character states measured using XAS are in fair agreement with the calculations (see Ref. [35], Discussion 1).

We have also confirmed that structural distortions alone, with $U=0$, do not lead to an insulating ground state. Thus, inclusion of a structural distortion in the presence of large 
$U$ is crucial for gap formation and MIT in $\mathrm{K}_{2} \mathrm{Cr}_{8} \mathrm{O}_{16}$. Hence, to gain further insight into the mechanism of MIT in $\mathrm{K}_{2} \mathrm{Cr}_{8} \mathrm{O}_{16}$, we performed calculations for different $U$ values in the range of $2-4 \mathrm{eV}$. Since the underlying electronic interaction strengths cannot be kept fixed as $U$ is varied in GGA $+U$ calculations, we supplemented the $\mathrm{GGA}+U$ results with those from parametrized $\mathrm{HF}+U$ calculations. Figure 3(c) shows the obtained band-gap values as a function of $U$ for the GGA $+U$ and $\mathrm{HF}+U$ calculations. The calculations were carried out for the tetramerized structure with and without polaronic distortions (see Ref. [35], Figs. S1 and S2), and the results are summarized in Fig. 3(c). The GGA $+U$ results indicate that the gap sets in at about $U \sim 2 \mathrm{eV}$ for both types of distortions and gradually increases as a function of $U$. The gaps are always smaller for the tetramerized structure compared to the tetramerized structure with polaronic distortions. This trend is also seen in the $\mathrm{HF}+U$ calculations. The energy stability was calculated in each case, and the tetramerized state was found to be the ground state above a $U$ of $3 \mathrm{eV}$. We also carried out calculations as a function of the magnitude of the distortion (see Ref. [35], Method 1). The results are shown in Fig. S3 of Ref. [35] and indicate that the band gap depends on the magnitude of distortion.

\section{Core-level spectra}

Figure 4(a) shows the $\mathrm{Cr}$ 1s core-level spectra obtained at $T=20,80,150$, and $250 \mathrm{~K}$, using HAXPES $(\mathrm{h} \nu=7935 \mathrm{eV})$, which is a bulk sensitive probe of the electronic structure [42]. The spectral shapes systematically evolve with temperature. At $T=20 \mathrm{~K}$, as shown in Fig. 4(b), the $\mathrm{Cr} 1 \mathrm{~s}$ spectrum shows a main peak at $5989.5 \mathrm{eV}$ and a shoulder structure at $5990.9 \mathrm{eV}$. In addition, weak features are seen at 5995- and 6001.5-eV binding energies. The $T=20 \mathrm{~K}$ spectrum could be simulated by using the $\mathrm{Cr}^{4+}$ and $\mathrm{Cr}^{3+}$ spectra [Fig. 4(b)] calculated using many-body cluster calculations (see Ref. [35], Method 2 for details), and adding them in the ratio of $3: 1$, corresponding to the nominal valence of 3.75 as per the chemical formula of $\mathrm{K}_{2} \mathrm{Cr}_{8} \mathrm{O}_{16}$. This is surprising because the x-ray diffraction results at $T=20 \mathrm{~K}$, in combination with the valence-bond sum analysis, indicated that the valency of all $\mathrm{Cr}$ atoms is the same, thus ruling out $\mathrm{Cr}^{4+}$ and $\mathrm{Cr}^{3+}$ states. Since photoemission is a very fast process with a core-hole lifetime of $10^{-15} \mathrm{sec}$, the only way we can then understand the spectroscopic results is that the core-hole excitation results in dynamic $\mathrm{Cr}^{4+}$ and $\mathrm{Cr}^{3+}$ valence fluctuations in $\mathrm{K}_{2} \mathrm{Cr}_{8} \mathrm{O}_{16}$. Table 1 shows the ground-state contributions to the $\mathrm{Cr}^{3+}$ and $\mathrm{Cr}^{4+}$ spectra. We obtain comparable $d^{n} \underline{L}^{0}\left(\equiv d^{n}\right)$ and $d^{n+1} \underline{L}^{1}$ ( $\underline{L}^{1}$ : ligand hole) character contributions for the $\mathrm{Cr}^{\frac{L^{3}}{+}}$ and $\mathrm{Cr}^{4+}$ spectra. The estimated charge-transfer energy $\Delta=2.5-3.0 \mathrm{eV}$, and it is smaller than the estimated
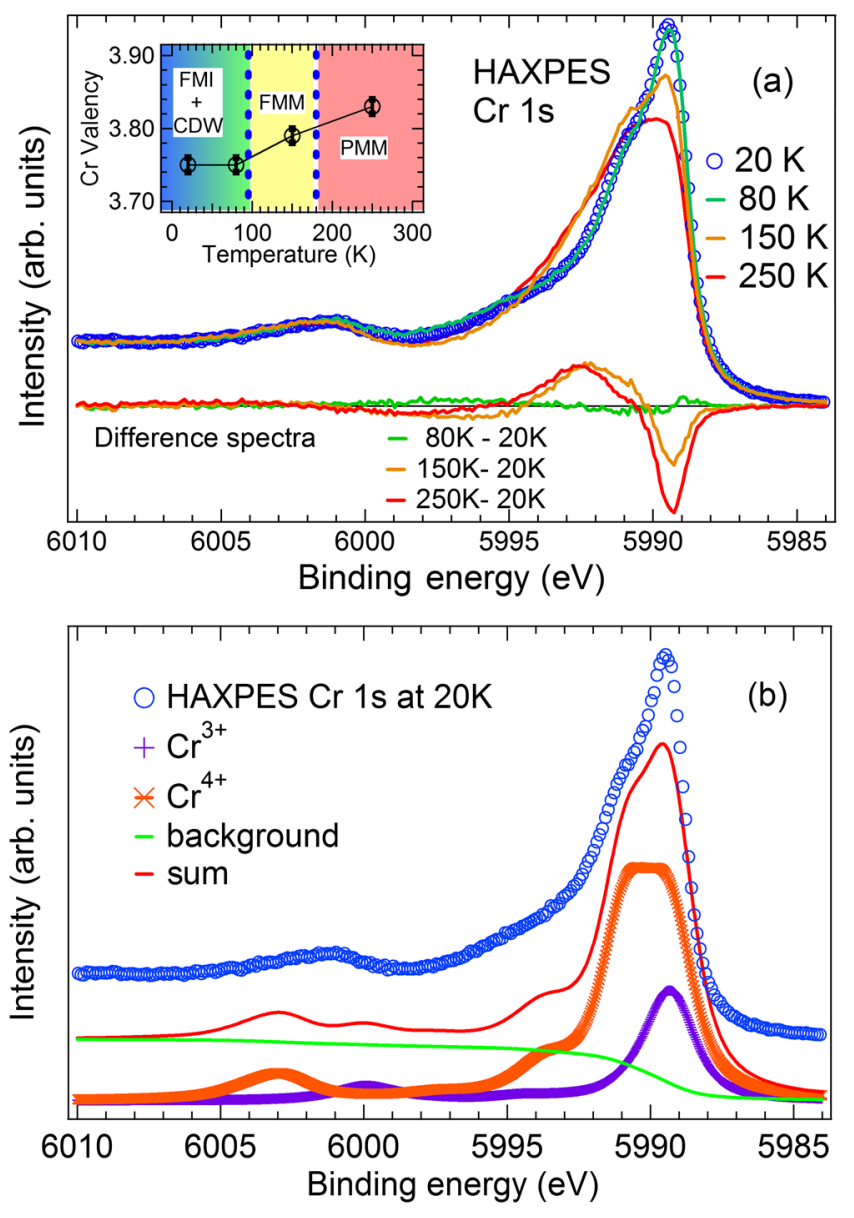

FIG. 4. Cr 1s core-level spectra of $\mathrm{K}_{2} \mathrm{Cr}_{8} \mathrm{O}_{16}$. (a) $T$-dependent $\mathrm{Cr}$ 1s core-level spectra obtained using HAXPES show clear changes across the paramagnetic-metal (PMM)-to-ferromagneticmetal (FMM) transition, but negligible change is seen between $80 \mathrm{~K}$ and $20 \mathrm{~K}$ in the Peierls ferromagnetic-insulating phase. The observation indicates that the average $\mathrm{Cr}$ valence gets pinned to $\mathrm{Cr}^{+3.75}$ in the Peierls phase. The inset shows the estimated average $\mathrm{Cr}$ valency as a function of $T$. (b) The $T=20-\mathrm{K}$ spectrum can be synthesized using the $\mathrm{Cr}^{4+}$ and $\mathrm{Cr}^{3+}$ spectra calculated using a cluster model and added in a ratio of $3: 1$, representing the average valency.

on-site Coulomb energy $U \sim 4 \mathrm{eV}$, indicating $\mathrm{K}_{2} \mathrm{Cr}_{8} \mathrm{O}_{16}$ is a charge-transfer material (see Ref. [35], Method 2). The experimental $\mathrm{K}_{2} \mathrm{Cr}_{8} \mathrm{O}_{16}$ XAS spectrum could also be simulated by combining the reference $\mathrm{Cr}^{4+}$ and $\mathrm{Cr}^{3+}$ L-edge XAS spectra in the ratio $3: 1$, confirming the nominal valence of $\mathrm{Cr}^{+3.75}$ (see Ref. [35], Discussion 1).

When increasing $T$, the spectra show a systematic broadening on the higher binding energy side of the main peak, and the shoulder and main peak merge to become a broad asymmetric peak. This behavior is in contrast to typical charge ordering or CDW transitions for which one usually obtains a narrow peak shape in the high- $T$ metallic phase that becomes broader in the low- $T$ insulating phase. In fact, canonical CDW systems show a $T$-dependent splitting, which increases when decreasing $T$ [43]. 
TABLE I. Ground-state contributions obtained for $\mathrm{Cr}^{3+}$ and $\mathrm{Cr}^{4+}$ core-level spectra shown in Fig. 4(b).

\begin{tabular}{lrr}
\hline \hline Configuration & $\mathrm{Cr}^{3+}$ & $\mathrm{Cr}^{4+}$ \\
\hline$d^{n} \underline{L}^{0}$ & $39.7 \%$ & $29.4 \%$ \\
$d^{n+1} \underline{L}^{1}$ & $42.0 \%$ & $27.7 \%$ \\
$d^{n+2} \underline{L^{2}}$ & $9.0 \%$ & $5.5 \%$ \\
$d^{n+1} \underline{C}$ & $6.5 \%$ & $25.7 \%$ \\
\hline \hline
\end{tabular}

Instead, the present spectra show a narrower width in the low-temperature Peierls insulating phase. As shown in the lower part of Fig. 4(a), the difference spectra plotted with respect to the $20-\mathrm{K}$ spectrum show systematic changes on going from $250 \mathrm{~K}$ to $150 \mathrm{~K}$ and also from $150 \mathrm{~K}$ to $80 \mathrm{~K}$. Surprisingly, the spectra show negligible change from $80 \mathrm{~K}$ to $20 \mathrm{~K}$. This indicates that the dynamic valence evolution occurring at high $T$ stops changing as a function of $T$ and gets pinned to an average value of $\mathrm{Cr}^{+3.75}$ in the ferromagnetic-insulating phase. In terms of the dynamic valence fluctuations, the $T$-dependent changes can be interpreted as a reduction in $\mathrm{Cr}^{3+}$ spectral intensity and an increase of $\mathrm{Cr}^{4+}$ spectral intensity when increasing $T$ since the $\mathrm{Cr}^{4+}$ states alone contribute to the shoulder structure at a binding energy of about $5992 \mathrm{eV}$ [Fig. 4(b)]. The $T=250-\mathrm{K}$ spectrum in Fig. 4(a) is dominated by the $\mathrm{Cr}^{4+}$, and this is confirmed by the calculated $\mathrm{Cr}^{4+}$ spectrum shown in Fig. 4(b). Compared to the 20-K spectrum, we estimate an increase in $\mathrm{Cr}^{4+}$ at the expense of $\mathrm{Cr}^{3+}$ content of about $4 \%$ and $8.5 \%$ at $150 \mathrm{~K}$ and $250 \mathrm{~K}$, respectively. In contrast, the spectra change by less than $1 \%$ between $20 \mathrm{~K}$ and $80 \mathrm{~K}$, which corresponds to the accuracy of estimating the valence change. The estimated average $\mathrm{Cr}$ valency as a function of $T$ is shown in the inset to Fig. 4(a). In addition, we have also carried out a very similar analysis for the $\mathrm{Cr}$ $2 p$ HAXPES spectra (see Ref. [35], Discussion 2) measured as a function of $T$, and we could obtain very consistent results with the $\mathrm{Cr} 1 s$ HAXPES spectra, thereby validating the results. It is noted that the assignment of $\mathrm{Cr}^{3+}$ and $\mathrm{Cr}^{4+}$-like features to dynamic fluctuations in the presence of a core hole, and its $T$ dependence, are also consistent with the observations that the $\mathrm{Cr} 3 d$ DOS do not show well-separated $\mathrm{Cr}^{3+}$ and $\mathrm{Cr}^{4+}$-like features, and the absence of $T$-dependent changes over large energy scales [see Figs. 2(a) and 2(b)].

\section{Metal-insulator transition using laser photoemission spectroscopy}

To confirm and quantify the gap formation across the MIT in $\mathrm{K}_{2} \mathrm{Cr}_{8} \mathrm{O}_{16}$, we carried out high-resolution laser PES measurements as a function of $T$. VB spectra were recorded in the temperature range $20-140 \mathrm{~K}$ and normalized for area under the curve, as presented in Fig. 5(a). A broad feature centered at about $0.2-\mathrm{eV} \mathrm{BE}$ is observed. On close inspection of the low-energy-scale electronic structure in the vicinity of $\mathrm{E}_{F}$, we observe a clear Fermi edge at $T=$ $140 \mathrm{~K}$ (see inset), confirming the metallic nature of $\mathrm{K}_{2} \mathrm{Cr}_{8} \mathrm{O}_{16}$. Upon decreasing $T$, a systematic sharpening and shift of the leading edge is observed in the spectra [Fig. 5(b)], indicating depletion of electronic states at and close to $\mathrm{E}_{F}$. This clear signature of opening of the gap at $\mathrm{E}_{F}$ confirms the low- $T$ insulating nature of $\mathrm{K}_{2} \mathrm{Cr}_{8} \mathrm{O}_{16}$ in accordance with resistivity measurements [9].

In order to estimate the $T$ dependence of the gap $[G(T)]$, we extracted the changes in the DOS at and near $\mathrm{E}_{F}$ by a symmetrization analysis of the normalized spectra of Fig. 5(b). The results of such an analysis are presented in Fig. 5(c). Evolution from a flat featureless behavior at $140 \mathrm{~K}$ (metallic phase) to a gap (or, more precisely, a pseudogap) at low $T$ is clearly seen in Fig. 5(c). The energy scale over which the spectral weight depletion occurs (in comparison to the $140-\mathrm{K}$ spectrum) is taken as an estimate of the total gap $[2 G(T)]$ in the occupied and unoccupied density of states, and its variation with $T$ is plotted in Fig. 5(d). For comparison, we also carried out an analysis of dividing the normalized spectra with the resolutionconvoluted Fermi-Dirac function, and estimated the gap. While this procedure is valid up to $5 k_{B} T$ above $\mathrm{E}_{F}$ [44] and gives an asymmetric gap, the symmetrization procedure is independent of temperature effects. The results of both the analyses are fairly consistent, as shown in Fig. 5(d). We estimate an error bar of $\pm 5 \mathrm{meV}$ for each data point. The energy-scale of the gap is quite small $[2 G(T=20 \mathrm{~K}) \sim$ $35 \pm 5 \mathrm{meV}$ ], and it reflects the subtle changes occurring in the low- $T$ electronic structure of $\mathrm{K}_{2} \mathrm{Cr}_{8} \mathrm{O}_{16}$. In the absence of any theoretical framework for describing a $T$-dependent FM-metal-to-FM-insulator transition, we compared the gap $2 G(T)$ vs $T$ plot with the well-known BCS-type gap behavior [23,24]. A satisfactory match is obtained with the calculated $2 G(0) \sim 3.5\left(k_{B} T_{\mathrm{MI}}\right) \sim 30 \mathrm{meV}$, as seen in Fig. 5(d). While more data points would have helped to make better agreement with the calculated curve, consistency of the present data with a BCS-type dependence of the gap suggests a second-order nature of the MIT in $\mathrm{K}_{2} \mathrm{Cr}_{8} \mathrm{O}_{16}$. This also links with the fact that an activation energy gap could not be extracted nor could any hysteresis be observed near $95 \mathrm{~K}$ in the resistivity measurements [9]. It is also consistent with the BCS-like $T$ dependence of the weak superstructure observed in diffraction experiments $[15,16]$. Furthermore, a close match of the gap magnitude with the BCS value using $T_{\mathrm{MI}}$ confirms a weak-coupling gap formation in the presence of strong $U$.

Thus, the strong $U$ sets up a low DOS at $\mathrm{E}_{F}$ but does not affect the gap energy scale in $\mathrm{K}_{2} \mathrm{Cr}_{8} \mathrm{O}_{16}$. Our firstprinciples calculations, in combination with the experimentally obtained value of $U$, the $T$-dependent gap, and the known $T$-dependent superstructure [15], indicate that $\mathrm{K}_{2} \mathrm{Cr}_{8} \mathrm{O}_{16}$ exhibits strong correlations with a charge-transfer ground state and valence evolution which gets pinned in the Peierls insulating phase. Furthermore, since the ground state 

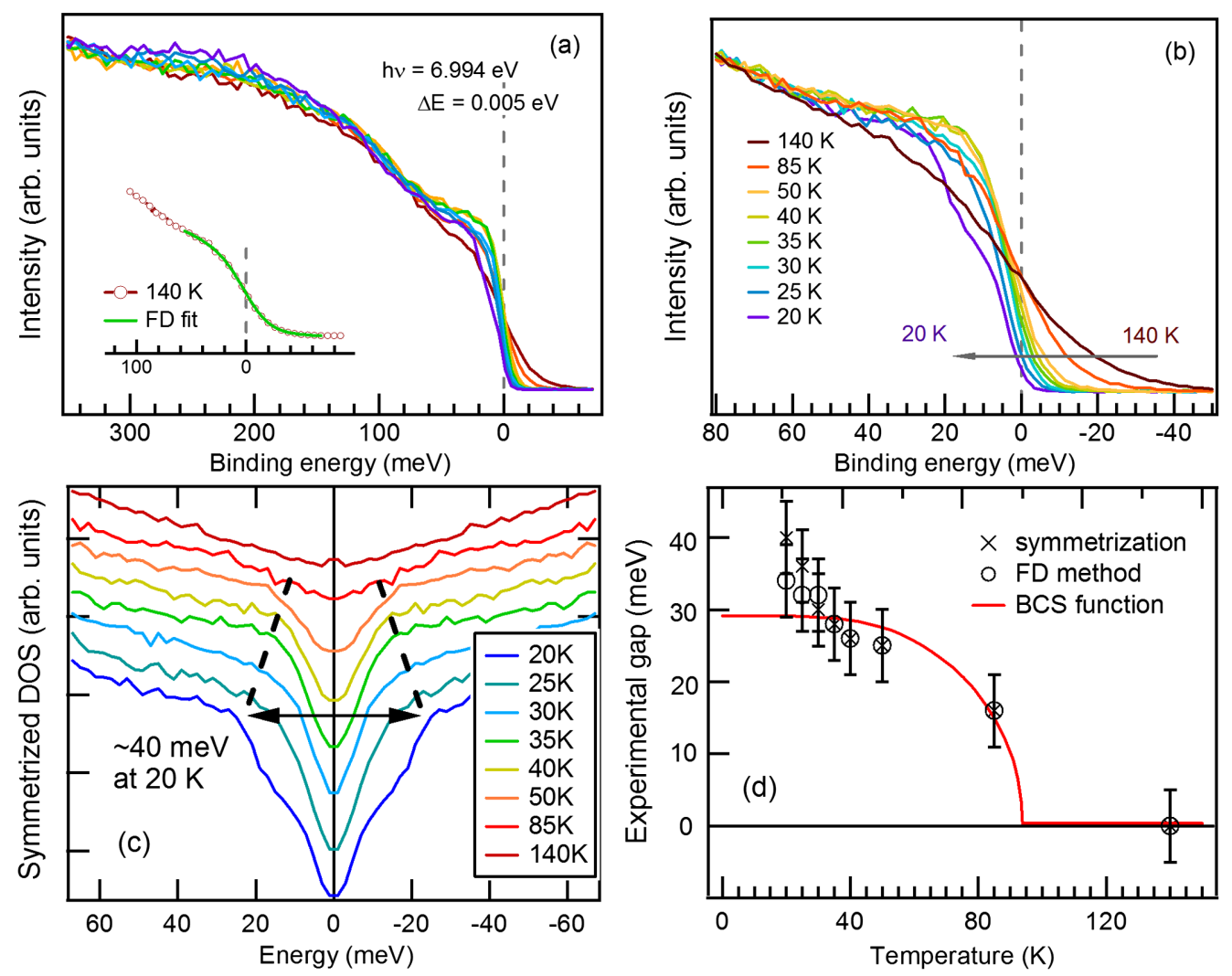

FIG. 5. Temperature-dependent laser photoemission of $\mathrm{K}_{2} \mathrm{Cr}_{8} \mathrm{O}_{16}$. (a) $T$-dependent $\mathrm{VB}$ spectra at and near $\mathrm{E}_{F}$, obtained using laser PES. The 0.2-eV feature is evident; the inset shows resolution-convoluted Fermi-Dirac (FD) function fit to the 140-K spectrum confirming a metallic Fermi edge. (b) The spectral intensity at $\mathrm{E}_{F}$ gets depleted gradually when decreasing $T$, indicative of a $T$-dependent gap formation. (c) DOS obtained upon symmetrizing the VB spectra at each $T$. (d) The gap vs $T$ shows a BCS-like dependence, suggestive of a second-order phase transition.

has significant $d^{n+1} \underline{L}^{1}$ for the $\mathrm{Cr}^{3+}$ and $\mathrm{Cr}^{4+}$ states, the pinning of valence fluctuations suggests that the Peierls insulating phase most likely involves excitations of the type $d^{n+1} \underline{L}^{1}+d^{n+1} \underline{L}^{1} \leftrightarrow d^{n+1} \underline{L}^{2}+d^{n+1} \underline{L}^{0}$; i.e., the charge fluctuations involve ligand holes, as was recently discussed for the nickelates [45]. Thus, while the Mott-Peierls picture describes spectral weight changes over large energy scales (about 1-2 eV), as has been discussed for the MIT in $\mathrm{VO}_{2}$ [18-20,46], and very recently, also for the MIT in related hollandites, $\mathrm{K}_{2} \mathrm{~V}_{8-x} \mathrm{Ti}_{x} \mathrm{O}_{16}$ [47], the present spectroscopy results with a low-energy-scale BCS-like $T$-dependent gap rule out a Mott-Peierls transition in $\mathrm{K}_{2} \mathrm{Cr}_{8} \mathrm{O}_{16}$, in contrast with such a proposition made in a very recent GGA $+U$ study [17]. Our results of $\mathrm{K}_{2} \mathrm{Cr}_{8} \mathrm{O}_{16}$ are summarized in Fig. 6: It is a strongly correlated charge-transfer FM halfmetal in the high- $T$ phase, which undergoes a low-energyscale BCS-like $T$-dependent gap formation in the low- $T$ FM-insulating phase.

A comparison with the typical Mott-Hubbard picture associated with a half-filled band is meaningful: The firstorder Mott-Hubbard transition results in an insulating phase with the lowest-energy excitation involving a $U$ between the majority and minority spin states and is

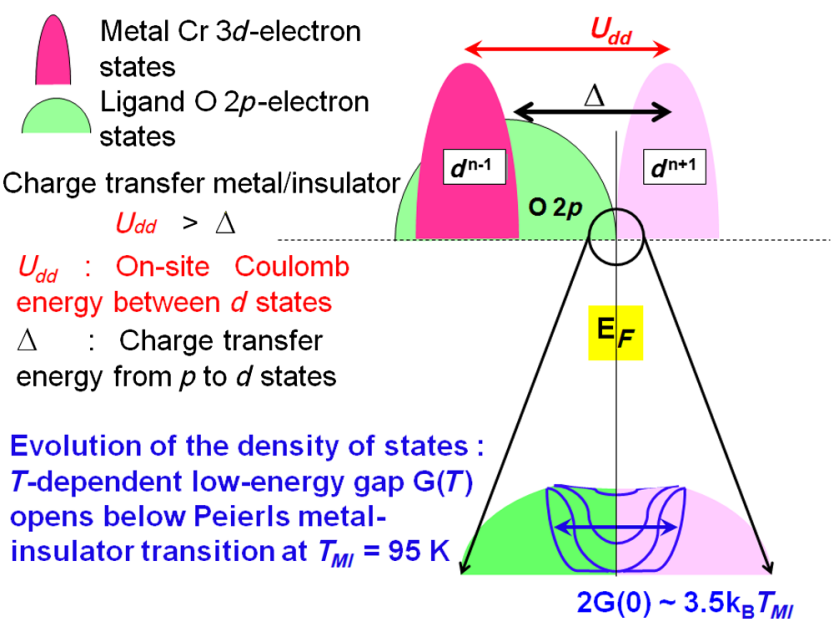

FIG. 6. Schematic electronic structure of $\mathrm{K}_{2} \mathrm{Cr}_{8} \mathrm{O}_{16} \cdot \mathrm{K}_{2} \mathrm{Cr}_{8} \mathrm{O}_{16}$ exhibits a charge-transfer-type half-metallic ground state with $U>\Delta$ in the high- $T$ ferromagnetic metal phase, as shown in the top panel. On reducing $T$, it undergoes a Peierls metal-insulator transition below $T_{\mathrm{MI}}=95 \mathrm{~K}$, with a gradual depletion of spectral weight at and near $\mathrm{E}_{F}$, consistent with a second-order BCS-like temperature-dependent gap (lower panel). 
described by an excitation of the type $2 d^{n} \rightarrow d^{n-1}+d^{n+1}$. Thus, the Mott-Hubbard transition naturally leads to an antiferromagnetic-insulating ground state. For the MottPeierls transition, the first-order structural distortion gets coupled to the formation of a nonmagnetic singlet state but still involves $U$ as the lowest-energy excitation [18-20]. For the present case of a nonintegral occupancy (2.25 $d$-electrons/Cr) and the half-metallic ferromagnetism in the $t_{2 g}$ up-spin band, the $U$ helps in defining the half-metallic ferromagnetism but also sets up a pseudogap in the $t_{2 g}$ upspin band. The pseudogap then undergoes a gradual gapping due to the structural distortion, but the lowestenergy excitation is within the $t_{2 g}$ up-spin band; hence, the ferromagnetism effectively favors a regular second-order Peierls transition.

\section{CONCLUSIONS}

In conclusion, we have carried out electronic structure studies of $\mathrm{K}_{2} \mathrm{Cr}_{8} \mathrm{O}_{16}$ across the sequential paramagneticmetal-to-FM-metal and FM-metal-to-FM-insulating transitions. Res-PES across $\mathrm{Cr} 2 p-3 d$ threshold indicates the presence of strong electron correlations $(U \sim 4.2 \mathrm{eV})$. First-principles GGA $+U$ and $\mathrm{HF}+U$ calculations show that a combination of large $U$ and small structural distortions are responsible for driving $\mathrm{K}_{2} \mathrm{Cr}_{8} \mathrm{O}_{16}$ insulating while maintaining FM order. Core-level spectra indicate clear $T$-dependent evolution of dynamic valence fluctuations at high temperatures, which get pinned in the lowtemperature Peierls phase of $\mathrm{K}_{2} \mathrm{Cr}_{8} \mathrm{O}_{16}$ to the nominal valence of $\mathrm{Cr}^{+3.75}$. The laser-PES spectra near $\mathrm{E}_{F}$ show systematic depletion in the DOS on lowering the temperature below $T_{\mathrm{MI}}$, and this yields a small gap (about $35 \mathrm{meV}$ at $20 \mathrm{~K}$ ), consistent with a weak-coupling BCS-like behavior with $2 G(0) \sim 3.5 \mathrm{k}_{B} T_{\mathrm{MI}}$.

\section{ACKNOWLEDGMENTS}

P. A. B acknowledges support from the Japan Society for Promotion of Science (JSPS). The synchrotron radiation experiments were performed at BL17SU and BL29XU, SPring-8, with the approval of RIKEN (Proposals No. 20091271 and No. 20100057). P. M. and M. L. acknowledge the support of the DST-DAAD personnel exchange programme (Project No. 55517034). Y. U. thanks JSPS for the Grant-in-Aid for Scientific Research (No. 19052004, No. 22244041, and No. 22104011). A. C. thanks JSPS for a Grant-in-Aid for Scientific Research (Challenging Exploratory Research Project No. 15K13526).

[1] A. Georges, G. Kotliar, W. Krauth, and M. J. Rozenberg, Dynamical Mean-Field Theory of Strongly Correlated Fermion Systems and the Limit of Infinite Dimensions, Rev. Mod. Phys. 68, 13 (1996).
[2] M. Imada, A. Fujimori, and Y. Tokura, Metal-Insulator Transitions, Rev. Mod. Phys. 70, 1039 (1998).

[3] D. B. McWhan and J. P. Remeika, Metal-Insulator Transition in $\left(\mathrm{V}_{1-x} \mathrm{Cr}_{x}\right)_{2} \mathrm{O}_{3}$, Phys. Rev. B 2, 3734 (1970).

[4] N. S. Rogado, J. Li, A. W. Sleight, and M. A. Subramanian, Magnetocapacitance and Magnetoresistance near Room Temperature in a Ferromagnetic Semiconductor: $\mathrm{La}_{2} \mathrm{NiMnO}_{6}$, Adv. Mater. 17, 2225 (2005).

[5] H. Das, U. V. Waghmare, T. Saha-Dasgupta, and D. D. Sarma, Electronic Structure, Phonons, and Dielectric Anomaly in Ferromagnetic Insulating Double Perovskite, Phys. Rev. Lett. 100, 186402 (2008).

[6] D. Choudhury, P. Mandal, R. Mathieu, A. Hazarika, S. Rajan, A. Sundaresan, U. V. Waghmare, R. Knut, O. Karis, P. Nordblad, and D. D. Sarma, Near-Room-Temperature Colossal Magnetodielectricity and Multiglass Properties in Partially Disordered $\mathrm{La}_{2} \mathrm{NiMnO}_{6}$, Phys. Rev. Lett. 108, 127201 (2012).

[7] V. Pardo and W. E. Pickett, Electron Confinement, Orbital Ordering, and Orbital Moments in $d^{0}-d^{1}$ Oxide Heterostructures, Phys. Rev. B 81, 245117 (2010).

[8] L. F. J. Piper, A. DeMasi, S. W. Cho, A. R. H. Preston, J. Laverock, K. E. Smith, K. G. West, J. W. Lu, and S. A. Wolf, Soft X-ray Spectroscopy of the Ferromagnetic Insulator $\mathrm{V}_{0.82} \mathrm{Cr}_{0.18} \mathrm{O}_{2}$, Phys. Rev. B 82, 235103 (2010).

[9] K. Hasegawa, M. Isobe, T. Yamauchi, H. Ueda, J. I. Yamaura, H. Gotou, T. Yagi, H. Sato, and Y. Ueda, Discovery of Ferromagnetic-Half-Metal-to-Insulator Transition in $\mathrm{K}_{2} \mathrm{Cr}_{8} \mathrm{O}_{16}$, Phys. Rev. Lett. 103, 146403 (2009).

[10] M. Sakamaki, T. Konishi, and Y. Ohta, $\mathrm{K}_{2} \mathrm{Cr}_{8} \mathrm{O}_{16}$ Predicted as a Half-Metallic Ferromagnet: Scenario for a MetalInsulator Transition, Phys. Rev. B 80, 024416 (2009); Phys. Rev. B 82, 099903(E) (2010).

[11] P. Mahadevan, A. Kumar, D. Choudhury, and D. D. Sarma, Charge Ordering Induced Ferromagnetic Insulator: $\mathrm{K}_{2} \mathrm{Cr}_{8} \mathrm{O}_{16}$, Phys. Rev. Lett. 104, 256401 (2010).

[12] P. Mahadevan, K. Terakura, and D. D. Sarma, Spin, Charge, and Orbital Ordering in $\mathrm{La}_{0.5} \mathrm{Sr}_{1.5} \mathrm{MnO}_{4}$, Phys. Rev. Lett. 87, 066404 (2001).

[13] V. Ferrari, M. Towler, and P. B. Littlewood, Oxygen Stripes in $\mathrm{La}_{0.5} \mathrm{Ca}_{0.5} \mathrm{MnO}_{3}$ from Ab-Initio Calculations, Phys. Rev. Lett. 91, 227202 (2003).

[14] P. H. L. Sit, R. Car, M. H. Cohen, and A. Selloni, Simple, Unambiguous Theoretical Approach to Oxidation State Determination via First-Principles Calculations, Inorg. Chem. 50, 10259 (2011).

[15] T. Toriyama, A. Nakao, Y. Yamaki, H. Nakao, Y. Murakami, K. Hasegawa, M. Isobe, Y. Ueda, A. V. Ushakov, D. I. Khomskii, S. V. Streltsov, T. Konishi, and Y. Ohta, Peierls Mechanism of the Metal-Insulator Transition in Ferromagnetic Hollandite $\mathrm{K}_{2} \mathrm{Cr}_{8} \mathrm{O}_{16}$, Phys. Rev. Lett. 107, 266402 (2011).

[16] A. Nakao, Y. Yamaki, H. Nakao, Y. Murakami, K. Hasegawa, M. Isobe, and Y. Ueda, Observation of Structural Change in the Novel Ferromagnetic Metal-Insulator transition in $\mathrm{K}_{2} \mathrm{Cr}_{8} \mathrm{O}_{16}$, J. Phys. Soc. Jpn. 81, 054710 (2012).

[17] S. Kim, K. Kim, and B. I. Min, Structural Instability and the Mott-Peierls Transition in a Half-Metallic Hollandite: $\mathrm{K}_{2} \mathrm{Cr}_{8} \mathrm{O}_{16}$, Phys. Rev. B 90, 045124 (2014). 
[18] S. Biermann, A. Poteryaev, A. I. Lichtenstein, and A. Georges, Dynamical Singlets and Correlation-Assisted Peierls Transition in $\mathrm{VO}_{2}$, Phys. Rev. Lett. 94, 026404 (2005).

[19] M. W. Haverkort, Z. Hu, A. Tanaka, W. Reichelt, S. V. Streltsov, M. A. Korotin, V. I. Anisimov, H. H. Hsieh, H.-J. Lin, C. T. Chen, D. I. Khomskii, and L. H. Tjeng, OrbitalAssisted Metal-Insulator Transition in $\mathrm{VO}_{2}$, Phys. Rev. Lett. 95, 196404 (2005).

[20] T. C. Koethe, Z. Hu, M. W. Haverkort, C. SchusslerLangeheine, F. Venturini, N. B. Brookes, O. Tjernberg, W. Reichelt, H. H. Hsieh, H.-J. Lin, C. T. Chen, and L. H. Tjeng, Transfer of Spectral Weight and Symmetry across the Metal-Insulator Transition in $\mathrm{VO}_{2}$, Phys. Rev. Lett. 97, 116402 (2006).

[21] C. Weber, D. D. O'Regan, N. D. M. Hine, M. C. Payne, G. Kotliar, and P. B. Littlewood, Vanadium Dioxide: A PeierlsMott Metal-Insulator Stable against Disorder, Phys. Rev. Lett. 108, 256402 (2012).

[22] R. E. Peierls, Quantum Theory of Solids (Oxford University, London, 1995).

[23] G. Grüner, The Dynamics of Charge-Density Waves, Rev. Mod. Phys. 60, 1129 (1988).

[24] J. Bardeen, L. N. Cooper, and J. R. Schrieffer, Theory of Superconductivity, Phys. Rev. 108, 1175 (1957).

[25] J. Solyom, The Fermi Gas Model of One-Dimensional Conductors, Adv. Phys. 28, 201 (1979).

[26] M. Isobe, S. Koishi, N. Kouno, J. Yamaura, T. Yamauchi, H. Ueda, H. Gotou, T. Yagi, and Y. Ueda, Observation of Metal-Insulator Transition in Hollandite Vanadate, $\mathrm{K}_{2} \mathrm{~V}_{8} \mathrm{O}_{16}$, J. Phys. Soc. Jpn. 75, 073801 (2006).

[27] M. Isobe, S. Koishi, S. Yamazaki, J. Yamaura, H. Gotou, T. Yagi, and Y. Ueda, Substitution Effect on Metal-Insulator Transition of $\mathrm{K}_{2} \mathrm{~V}_{8} \mathrm{O}_{16}$, J. Phys. Soc. Jpn. 78, 114713 (2009).

[28] E. Şaşioğlu, C. Friedrich, and S. Blügel, Effective Coulomb Interaction in Transition Metals from Constrained Random-Phase Approximation, Phys. Rev. B 83, 121101 (2011).

[29] See http://www.flapw.de for details of the FLAPW method.

[30] F. Freimuth, Y. Mokrousov, D. Wortmann, S. Heinze, and S. Blügel, Maximally Localized Wannier Functions within the FLAPW Formalism, Phys. Rev. B 78, 035120 (2008).

[31] A. A. Mostofi, J. R. Yates, Y.-S. Lee, I. Souza, D. Vanderbilt, and N. Marzari, Wannier90: A Tool for Obtaining Maximally-Localised Wannier Functions, Comput. Phys. Commun. 178, 685 (2008).

[32] Y. Takata, M. Yabashi, K. Tamasaku, Y. Nishino, D. Miwa, T. Ishikawa, E. Ikenaga, K. Horiba, S. Shin, M. Arita, K. Shimada, H. Namatame, M. Taniguchi, H. Nohira, T. Hattori, S. Sodergren, B. Wannberg, and K. Kobayashi, Development of Hard X-ray Photoelectron Spectroscopy at BL29XU in SPring-8, Nucl. Instrum. Methods Phys. Res., Sect. A 547, 50 (2005).

[33] T. Ishikawa, K. Tamasaku, and M. Yabashi, HighResolution X-ray Monochromators, Nucl. Instrum. Methods Phys. Res., Sect. A 547, 42 (2005).
[34] T. Kiss, T. Shimojima, K. Ishizaka, A. Chainani, T. Togashi, T. Kanai, X.-Y. Wang, C.-T. Chen, S. Watanabe, and S. Shin, A Versatile System for Ultrahigh Resolution, Low Temperature, and Polarization Dependent Laser-Angle-Resolved Photoemission, Rev. Sci. Instrum. 79, 023106 (2008).

[35] See Supplemental Material at http://link.aps.org/ supplemental/10.1103/PhysRevX.5.041004 for details of the band structure calculations, model cluster calculations, and supporting theoretical and experimental results.

[36] S. Hüfner, S.-H. Yang, B. S. Mun, C. S. Fadley, J. Schäfer, E. Rotenberg, and S. D. Kevan, Observation of the TwoHole Satellite in $\mathrm{Cr}$ and $\mathrm{Fe}$ Metal by Resonant Photoemission at the 2p Absorption Energy, Phys. Rev. B 61, 12582 (2000).

[37] L. Galán, M. García, J. M. Ripalda, I. Montero, E. Roman, D. R. Batchelor, and P. R. Bressler, Resonant Photoemission in Cr Silicide at the Absorption Energy Cr 2p, Appl. Phys. Lett. 84, 4433 (2004).

[38] P. A. Bhobe, A. Chainani, M. Taguchi, T. Takeuchi, R. Eguchi, M. Matsunami, K. Ishizaka, Y. Takata, M. Oura, Y. Senba, H. Ohashi, Y. Nishino, M. Yabashi, K. Tamasaku, T. Ishikawa, K. Takenaka, H. Takagi, and S. Shin, Evidence for a Correlated Insulator to Antiferromagnetic Metal Transition in CrN, Phys. Rev. Lett. 104, 236404 (2010).

[39] P. A. Bhobe, A. Chainani, M. Taguchi, R. Eguchi, M. Matsunami, T. Ohtsuki, K. Ishizaka, M. Okawa, M. Oura, Y. Senba, H. Ohashi, M. Isobe, Y. Ueda, and S. Shin, Electronic Structure of an Antiferromagnetic Metal: $\mathrm{CaCrO}_{3}$, Phys. Rev. B 83, 165132 (2011).

[40] G. Kresse and J. Furthmuller, Effective Iterative Schemes for Ab Initio Total-Energy Calculations Using a Plane-Wave Basis Set, Phys. Rev. B 54, 11169 (1996).

[41] G. Kresse and J. Furthmuller, Efficiency of Ab-Initio Total Energy Calculations for Metals and Semiconductors Using a Plane-Wave Basis Set, Comput. Mater. Sci. 6, 15 (1996).

[42] A. X. Gray, C. Papp, S. Ueda, B. Balke, Y. Yamashita, L. Plucinski, J. Minar, J. Braun, E. R. Ylvisaker, C. M. Schneider, W. Pickett, H. Ebert, K. Kobayashi, and C. S. Fadley, Probing Bulk Electronic Structure with Hard X-ray Angle-Resolved Photoemission, Nat. Mater. 10, 759 (2011).

[43] H. P. Hughes and J. A. Scarfe, Site-Specific Photohole Screening in a Charge Density Wave, Phys. Rev. Lett. 74, 3069 (1995).

[44] T. Greber, T. J. Kreutz, and J. Osterwalder, Photoemission above the Fermi Level: The Top of the Minority $d$ Band of Nickel, Phys. Rev. Lett. 79, 4465 (1997).

[45] S. Johnston, A. Mukherjee, I. Elfimov, M. Berciu, and G. A. Sawatzky, Charge Disproportionation without Charge Transfer in the Rare-Earth Nickelates as a Possible Mechanism for the Metal-Insulator Transition, Phys. Rev. Lett. 112, 106404 (2014).

[46] S. Kim, K. Kim, C.-J. Kang, and B. I. Min, CorrelationAssisted Phonon Softening and the Orbital-Selective Peierls Transition in $\mathrm{VO}_{2}$, Phys. Rev. B 87, 195106 (2013).

[47] Y. Ishige, T. Sudayama, Y. Wakisaka, T. Mizokawa, H. Wadati, G. A. Sawatzky, T.Z. Regier, M. Isobe, and Y. Ueda, Interplay between Mott Physics and Peierls Physics in Hollandite-Type Vanadates with a Metal-Insulator Transition, Phys. Rev. B 83, 125112 (2011). 